\title{
The choriocarcinoma cell line BeWo: syncytial fusion and expression of syncytium-specific proteins
}

\author{
Kristina Orendi, Martin Gauster, Gerit Moser, Hamutal Meiri ${ }^{1}$ and Berthold Huppertz \\ Institute of Cell Biology, Histology and Embryology, Centre for Molecular Medicine, Medical University of Graz, \\ Harrachgasse 21/VII, 8010 Graz, Austria and ${ }^{1}$ Diagnostic Technologies Ltd, 20692 Yoqneam, Israel \\ Correspondence should be addressed to B Huppertz; Email: berthold.huppertz@medunigraz.at
}

\begin{abstract}
Fusion of the trophoblast-derived choriocarcinoma cell line BeWo can be triggered by forskolin. BeWo cells are regularly used as a cell culture model to mimic in vivo syncytialisation of placental villous trophoblast. The $\boldsymbol{\beta}$ subunit of human chorionic gonadotropin (CGB), placental alkaline phosphatase as well as placental protein 13 (PP13, LGALS13) are exclusively expressed in the syncytiotrophoblast of the human placenta, and CGB is commonly used as a marker of syncytial differentiation. Here we tested the hypothesis that syncytial fusion precedes CGB and LGALS13 expression in trophoblast-derived BeWo cells. BeWo cells were cultured for $48 \mathrm{~h}$ in the presence or absence of forskolin and varying concentrations of $\mathrm{H}-89$, a protein kinase $\mathrm{A}$ inhibitor that interferes with the forskolin-mediated pathway of syncytial fusion. LGALS13 and CGB expression were quantified by DELFIA and real-time PCR. Cell fusion was determined by morphological analysis and cell counting after immunofluorescence staining. In forskolin-stimulated BeWo cells that were hindered to fuse by treatment with $\mathrm{H}-89$, levels of CGB protein expression were not altered, while LGALS13 protein and mRNA expression decreased significantly to control levels without forskolin. The LGALS13 protein expression data coincided with a significant decrease in syncytial fusion, while CGB protein expression was unaffected by rates of cell fusion and proliferation. We postulate that CGB protein expression is not necessarily linked to syncytial fusion, and thus CGB should be used with great caution as a marker of BeWo cell fusion. Reproduction (2010) $\mathbf{1 4 0} 759-766$
\end{abstract}

\section{Introduction}

The choriocarcinoma cell line BeWo is the most extensively used cellular in vitro model to study villous trophoblast fusion. These cells show a low spontaneous fusion rate, which can be boosted by the addition of CAMP, its analogue 8-bromo-CAMP, or by adding forskolin (Wice et al. 1990). The latter increases intracellular cAMP levels by activating adenylyl cyclase. In turn, increased cAMP activates protein kinase A (PKA; Knerr et al. 2005), subsequently activating proteins such as the transcription factor glial cells missing 1 (GCM1). Target genes of GCM1 include the classical fusion peptide syncytin-1 (ERVWE1), which is directly involved in trophoblast fusion (Mi et al. 2000). Finally, proteins specific for the syncytiotrophoblast such as $\beta$ subunit of human chorionic gonadotropin (CGB) and placental protein 13 (PP13, LGALS13) are upregulated after syncytialisation (Fig. 1A).

The placental-specific LGALS13 (Than et al. 2004) is a member of the $\beta$-galactoside binding S-type galectin superfamily and is one of the promising biochemical markers to predict preeclampsia, a pregnancy-related syndrome (Spencer et al. 2007,
Huppertz et al. 2008). In humans, LGALS13 is only expressed in placental tissues, and within the villous trophoblast it can only be detected in the multinucleated syncytiotrophoblast (Huppertz et al. 2008, Than et al. 2009).

CGB and ERVWE1 are commonly used as markers for syncytiotrophoblast in primary trophoblast (Frendo et al. 2003) and BeWo cells (Butler et al. 2009). Other proteins that are often used as markers for syncytiotrophoblast are human placental lactogen (hPL; CSH1) (Gaspard et al. 1980, Pidoux et al. 2007) and human placental alkaline phosphatase (ALPP; Webb \& Todd 1988, Guilbert et al. 2002). Regarding CGB, former studies have already shown that BeWo cell fusion is not necessarily linked to $C G B$ mRNA expression (Lin et al. 1999). In a follow-up study, these authors showed cellular rather than syncytial CGB expression in ERV3-overexpressing cells (Lin et al. 2000).

To test the hypothesis that the expression of LGALS13 and CGB is indeed restricted to the syncytial form of the trophoblast-derived BeWo cells, we analysed fusion of BeWo cells and expression of several key players in the fusion process in response to forskolin treatment. 


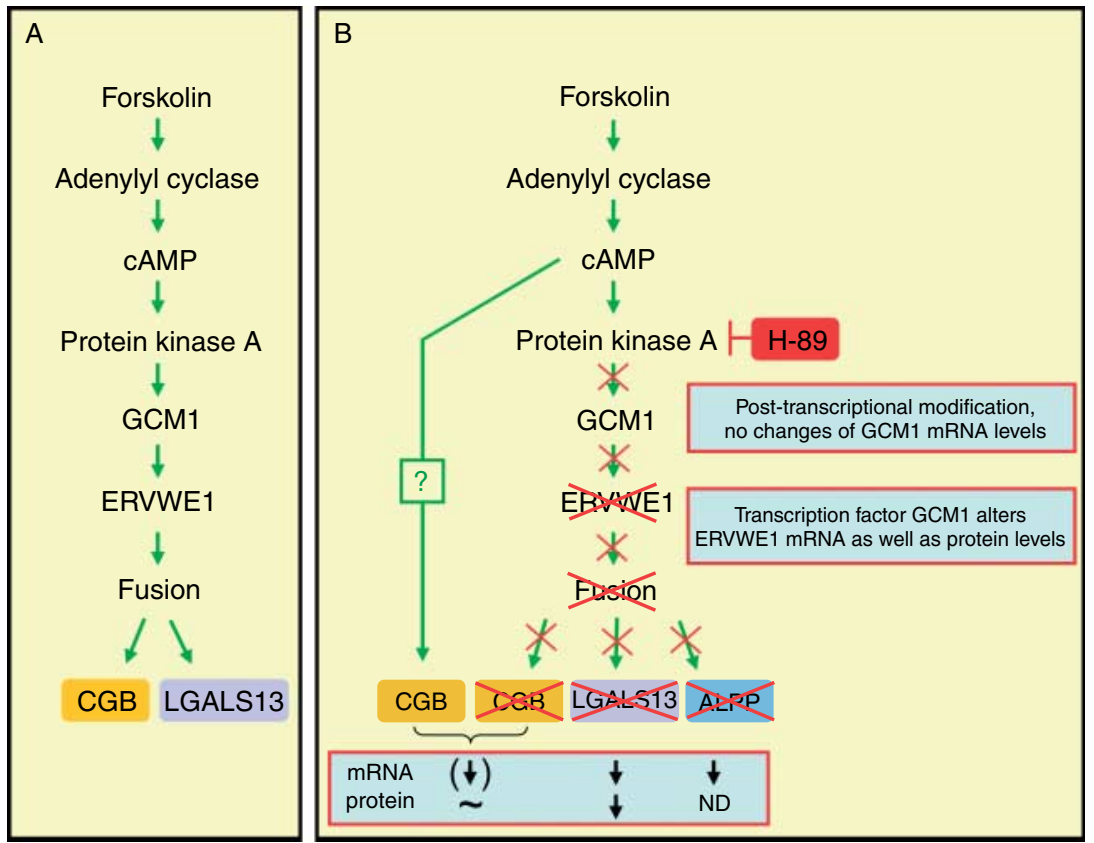

Figure 1 Proposed pathway of forskolin-mediated BeWo cell fusion. (A) Pathway of forskolin-mediated cell fusion and expression of CGB and LGALS13 as accepted so far. LGALS13 as well as CGB are only considered to be expressed after fusion of BeWo cells. (B) Proposed pathway of forskolin-mediated cell fusion of BeWo cells combined with the expression of GCM1, ERVWE1, CGB, LGALS13 and ALPP in the presence of $\mathrm{H}-89$ as derived from this study. LGALS13 protein expression is linked to fusion, while CGB protein expression is at least partially independent of syncytial fusion. Blockage of protein kinase A hinders cell fusion, LGALS13 mRNA and protein expression and ALPP mRNA expression, as marked with black arrows. Expression of CGB protein remains unchanged in the presence of $\mathrm{H}-89$, as marked with a tilde $(\sim)$, while $C G B$ mRNA expression is significantly downregulated compared with that in the presence of forskolin only but at the same time still remains significantly upregulated compared with that in the presence of DMSO, as marked with a black arrow in brackets. Protein expression of ALPP was not determined (ND).

\section{Results}

\section{H-89 inhibits protein expression of LGALS13 but not of $C G B$}

BeWo cells were tested for their ability to express LGALS13 and CGB at the protein level in the presence or absence of $20 \mu \mathrm{M}$ forskolin and in the presence or absence of various concentrations of $\mathrm{H}-89$. In the presence of DMSO, the expression of both proteins was low, while in the presence of forskolin the expression was upregulated up to 3.2-fold in the case of LGALS13 and up to 7.4-fold in the case of CGB (Fig. 2A and B). In the presence of forskolin, addition of $10 \mu \mathrm{M} \mathrm{H}-89$ (Davies et al. 2000), a cell permeable and potent inhibitor of PKA, reduced the expression of LGALS13 to levels to those observed in the absence of forskolin (Fig. 2A). Interestingly, the expression of CGB was not altered in the presence of up to $10 \mu \mathrm{M} \mathrm{H}$-89 (Fig. 2B). At a concentration of $1 \mu \mathrm{M}, \mathrm{H}-89$ had no effect on the expression of LGALS13 and CGB. Higher concentrations of $\mathrm{H}-89,30$ and $100 \mu \mathrm{M}$, were lethal for the cells.

Since a concentration of $10 \mu \mathrm{M} \mathrm{H}-89$ was most effective in our test system, this concentration was used for all further experiments.

\section{BeWo cell fusion is reduced in the presence of $\mathbf{H - 8 9}$}

Figure $3 \mathrm{~A}$ shows immunofluorescence staining of BeWo cells in the presence of $0.2 \% \mathrm{DMSO}, 20 \mu \mathrm{M}$ forskolin or $10 \mu \mathrm{M} \mathrm{H}-89$ in combination with $20 \mu \mathrm{M}$ forskolin. The DMSO-treated controls showed only very little CGB staining, and staining for fodrin (SPTAN1), a major component of the sub- $\alpha$-membranous cytoskeleton, revealed that the cells were mostly in the mononucleated stage (Fig. 3A, I-III). Treatment with forskolin upregulated differentiation and fusion of BeWo cells. This was visualised by plaques of CGB staining (Fig. 3A, IV and $\mathrm{VI}$ ) and absence of SPTAN1 staining in larger areas (Fig. 3A, V-VI). This staining pattern demonstrated syncytialisation, as cell border-associated SPTAN1 network was previously shown to be downregulated and degraded during trophoblast fusion (Gauster et al. 2010). In the presence of $\mathrm{H}-89$, individual BeWo cells stained strongly positive for CGB but remained in the mononucleated stage (Fig. 3A, VII and IX). The clear cell borders as visualised by SPTAN1 staining (Fig. 3A, VIIIIX) were similar to those visualised in the DMSO-treated control group. The double labelling showed that mononucleated cells were indeed expressing CGB (Fig. 3A, IX). 

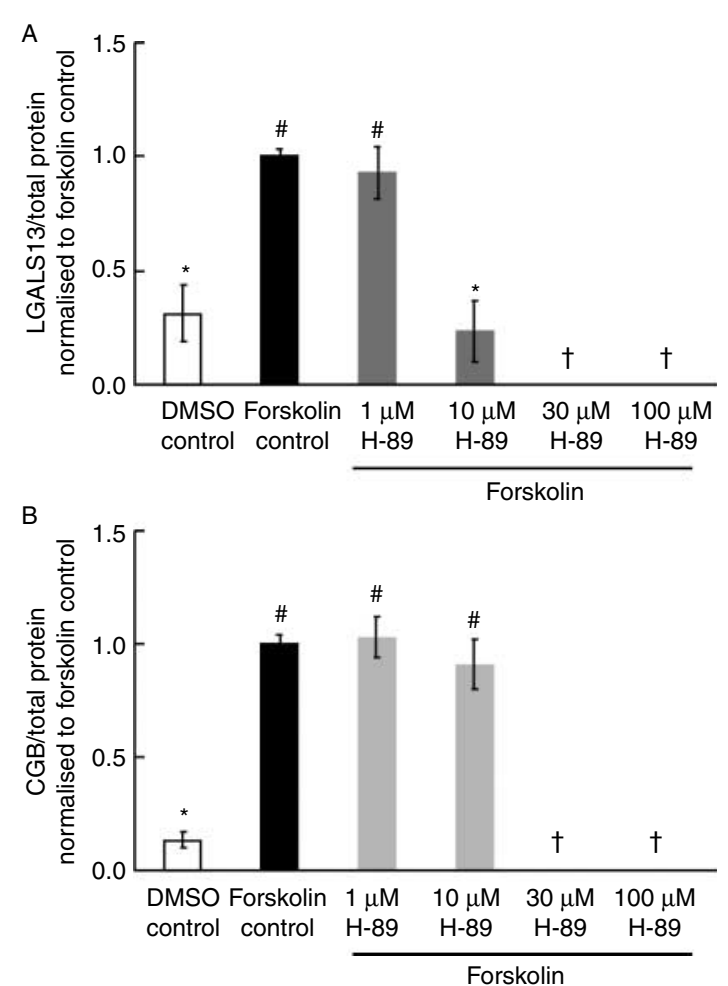

Figure 2 Effect of $\mathrm{H}-89$ and forskolin on LGALS13 (A) and CGB (B) expression in BeWo cells (48 h) (mean \pm s.D.). $\mathrm{H}-89$ treatment led to decreased LGALS13 protein levels (dark grey bars) at $10 \mu \mathrm{M}$, while CGB protein levels remained unaffected (light grey bars). At 30 and $100 \mu \mathrm{M} \mathrm{H}-89$, cells were no longer viable $(\dagger)$. All data were normalised to the forskolin control (black bars). Significant differences from the forskolin control are marked with an asterisk (*). Significant differences from the DMSO-treated controls (white bars) are marked with a hash $(\#)$. Data are obtained from nine independent experiments.

Quantification of BeWo cell fusion confirmed that forskolin induced cell fusion in these cells. While DMSO-treated controls showed a spontaneous fusion rate of about $4.9 \%$, forskolin treatment increased fusion events significantly to $50.5 \%$. The effect of forskolin was nearly diminished to a fusion rate of $11.0 \%$ in the presence of $10 \mu \mathrm{M} \mathrm{H}-89$ with a significant decrease of fusion events compared with the effect of forskolin only (Fig. 3B). Nevertheless, the fusion rate in the presence of $\mathrm{H}-89$ and forskolin was still significantly increased compared with that in the presence of DMSO, although there was a significant decrease towards a much lower fusion rate in the presence of $\mathrm{H}-89$ compared with that in the presence of forskolin.

\section{$\mathrm{H}-89$ reduces the forskolin-induced activation of mRNA expression of ERVWE1, LGALS13 and CGB in BeWo cells}

Following the proposed pathways of forskolin action as depicted in Fig. 1A, the mRNA expression of GCM1, ERVWE1, LGALS13 and CGB in BeWo cells was analysed. Furthermore, the mRNA expression of a commonly used marker for syncytialisation, $A L P P$, and of the sub-membranous cytoskeletal protein SPTAN1 were assessed in the presence of DMSO, forskolin or $\mathrm{H}-89$ in combination with forskolin by real-time RT-PCR (Fig. 4A-F). In the presence of forskolin, the mRNA expression of GCM1, ERVWE1, LGALS13, CGB and $A L P P$ were significantly upregulated compared with that in the presence of DMSO (Fig. 4A-E), while the mRNA expression of SPTAN1 decreased significantly when the cells were treated with forskolin (Fig. 4F). Addition of $\mathrm{H}-89$ to the culture medium in the presence of forskolin resulted in a significant decrease of ERVWE1, LGALS13 and $C G B$ (Fig. 4B-D) compared with that in the presence of forskolin. ERVWE1 and LGALS13 expression even dropped down to levels of DMSO-treated controls (Fig. 4B and C), while CGB levels still showed a 10 -fold increase compared with the levels of DMSOtreated controls (Fig. 4D). The mRNA expression of GCM1, ALPP and SPTAN1 was not significantly altered in the presence of $\mathrm{H}-89$ and forskolin compared with that in the presence of forskolin only, although a trend towards the expression level in the presence of DMSO could be observed in the case of ALPP (Fig. 4E and F).

\section{$\mathrm{H}-89$ keeps proliferation active in BeWo cells staining positive for $C G B$}

Immunofluorescence staining with antibodies against CGB and the proliferation marker Ki-67 (Gerdes et al. 1983) reveals that the vast majority of nuclei in the presence of DMSO stain positive for $\mathrm{Ki}-67$, indicating that these cells are proliferating (Fig. 5A). CGB expression in these cells is low. In the presence of forskolin, nuclei in CGB stain positive and multinucleated cell structures stain mainly negative for $\mathrm{Ki}-67$ (Fig. 5B). In the presence of $\mathrm{H}-89$ and forskolin, a great number of cells staining positive for CGB are still proliferating as shown by Ki-67-positive nuclei (Fig. 5C).

Data shown in Figs $2-5$ point out that the originally proposed, simplified pathway of forskolin-induced BeWo cell fusion and differentiation (Fig. 1A) needs updating as soon as inhibition of PKA by $\mathrm{H}-89$ comes into play. Figure $1 \mathrm{~B}$ shows a more complex attempt to explain the interrelation of several key steps in this forskolin-induced fusion cascade, pointing out that the regulation of CGB might follow an additional, PKA-independent pathway. Furthermore, mRNA data revealed that expression of genes is often differentially regulated than that of the respective proteins, as is the case for $\mathrm{CGB}$, for example. While at the protein level CGB expression in the presence of $\mathrm{H}-89$ and forskolin was not altered at all compared with that in the presence of forskolin (Fig. 2B), CGB mRNA expression decreased significantly from 35 -fold in the presence of forskolin to 10 -fold in the presence of forskolin and $\mathrm{H}-89$ compared with that in the presence of DMSO (Fig. 4D). 
A
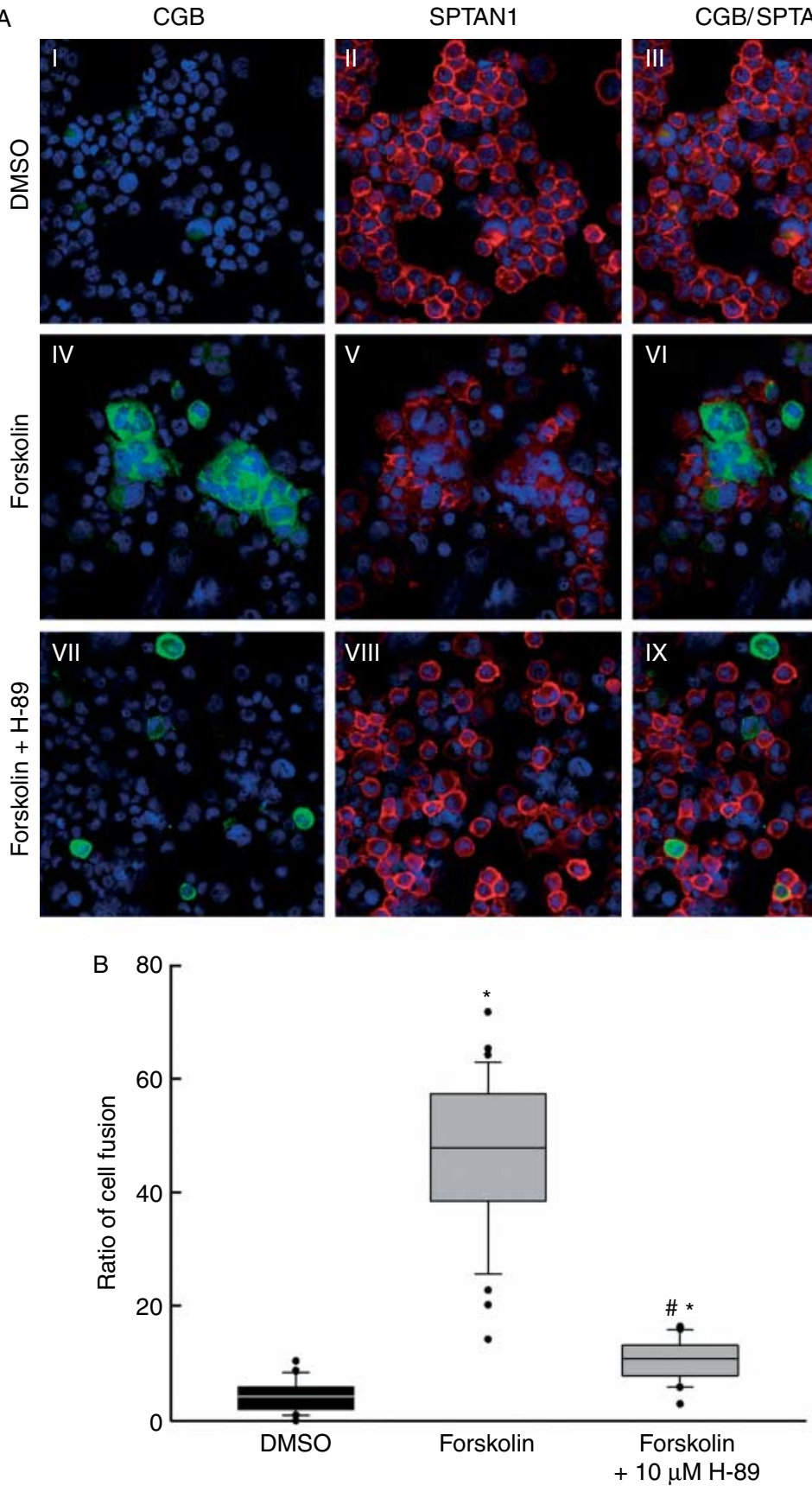

SPTAN1
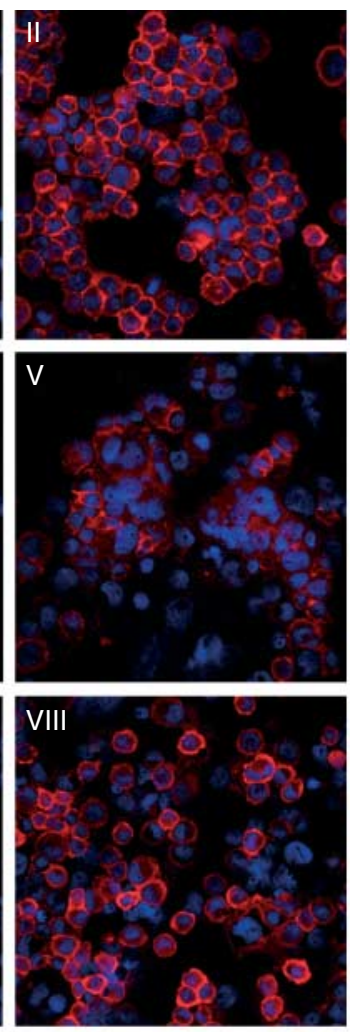

CGB/SPTAN1
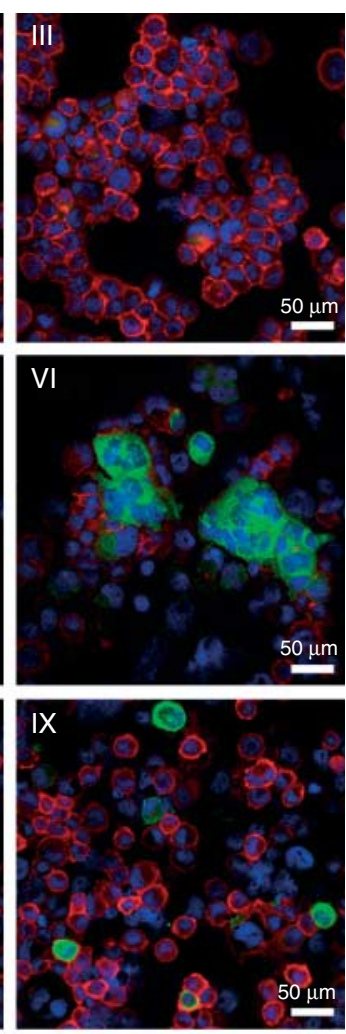

$+10 \mu \mathrm{M} \mathrm{H}-89$
Figure 3 Effect of the protein kinase $\mathrm{A}$ inhibitor $\mathrm{H}-89$ on BeWo cell fusion and CGB expression (48 $\mathrm{h}$ ). (A) Immunofluorescence staining for CGB (I, IV, VII: green) and SPTAN1 (II, V, VIII: red) with DAPI counterstaining (blue) was performed on BeWo cytospins as described in Materials and Methods. In the presence of $\mathrm{H}-89$, mononucleated cells stained strongly for $\mathrm{CGB}$, while the number of syncytia was reduced. (B) Fusion rates in the presence of DMSO, forskolin or H-89 and forskolin. Data are presented as box-and-whisker plot. Significant differences to DMSO-treated controls are marked with an asterisk $\left.{ }^{*}\right)$. Significant differences to forskolin-treated cells are marked with a hash $(\#)$. Data were obtained from three independent experiments.

\section{Discussion}

Syncytial fusion in vivo is one of the key events occurring during placental development. CGB is often described as a marker of syncytial differentiation (Butler et al. 2009), and in vitro studies using villous explants and isolated primary trophoblasts have revealed that CGB can indeed be used as marker for syncytialisation of primary trophoblast (Leisser et al. 2006). This is in clear agreement with a variety of other studies showing that CGB is upregulated in fusing BeWo cells as well (Butler et al. 2009). Different from CGB, the $\alpha$ subunit of human chorionic gonadotropin (CGA) has been described to be upregulated already prior to syncytial fusion (Gaspard et al. 1980). LGALS13 expression in BeWo cells and its dependency on BeWo cell fusion has not been shown before. Here, it was shown that the expression of CGB and LGALS13 was related to BeWo cell fusion.

According to the proposed pathway of forskolininduced cell fusion (Fig. 1A), the latter is mediated through an increase of CAMP, activation of PKA and of the transcription factor GCM1, finally activating ERVWE1. This results in BeWo cell fusion and the 

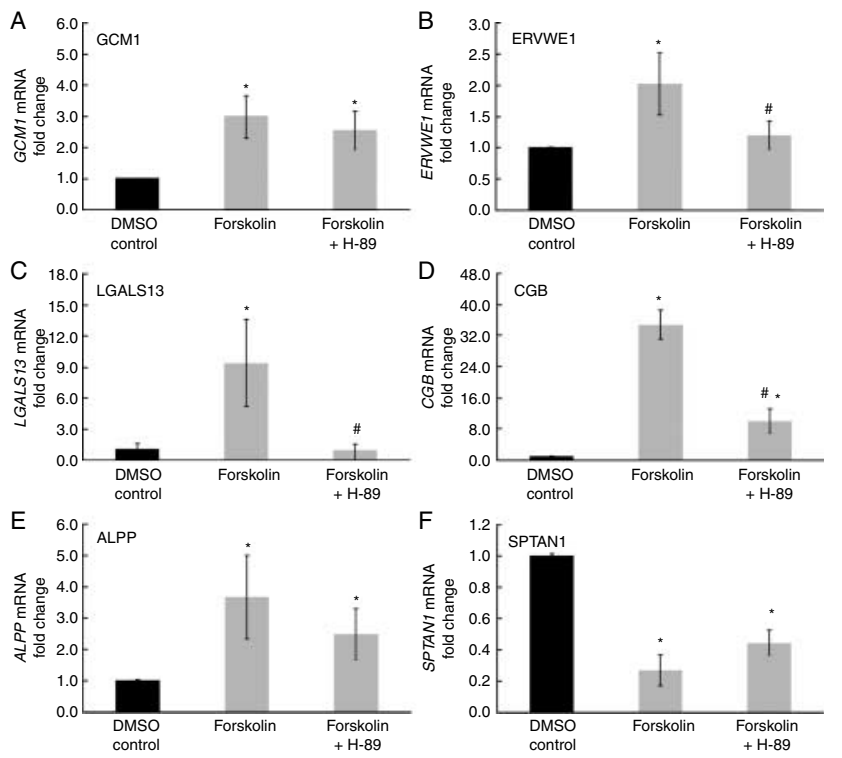

Figure 4 Effect of the protein kinase $\mathrm{A}$ inhibitor $\mathrm{H}-89$ on mRNA expression of GCM1, ERVWE1, LGALS13, CGB, ALPP and SPTAN1 $(48 \mathrm{~h})$. Quantitative real-time RT-PCR of BeWo cells in the presence of DMSO, forskolin and $\mathrm{H}-89$ for $48 \mathrm{~h}$ (mean \pm s.D.). Values represent fold changes relative to DMSO-treated controls (black bars). Significant differences to DMSO-treated controls are marked with an asterisk $(*)$, significant differences to forskolin-treated cells are marked with a hash $(\#)$. Data are obtained from three to four independent experiments.

subsequent protein expression of LGALS13 and CGB (Wice et al. 1990, Knerr et al. 2005, Schubert et al. 2008). Hence, the hypothesis was made that an inhibitor of PKA should thus block the whole pathway of fusion as well as the expression of both the proteins, CGB and LGALS13 (Keryer et al. 1998).

According to our data, this was true for syncytial fusion of BeWo cells and the expression of LGALS13. $\mathrm{H}-89$ is a blocker of PKA but inhibits several other kinases such as S6K, MSK1, PKA and ROCK2 as well (Davies et al. 2000, Leemhuis et al. 2002). In our setting, $\mathrm{H}-89$ interfered with the forskolin-mediated cAMPdependent pathway of syncytial fusion, and the subsequent LGALS13 expression was completely blocked with no differences of LGALS13 expression level compared with DMSO-treated controls (Fig. 2A).

Interestingly, this was not the case for CGB. In BeWo cells, CGB was not only expressed in multinucleated syncytia but also in mononucleated cells hindered to undergo fusion via $\mathrm{H}-89$ treatment (Fig. 3A, IX). The forskolin-induced protein expression of $\mathrm{CGB}$ was not blocked by the PKA inhibitor with no significant differences to forskolin controls in the absence of $\mathrm{H}-89$ (Fig. 2B). Thus, CGB expression seems to follow a second, PKA-independent pathway (Fig. 1B).

LGALS13 protein data after $\mathrm{H}-89$ treatment could be confirmed at the mRNA level (Fig. 4C). This was not the case for CGB as $C G B$ mRNA was affected by inhibition of PKA, even though it was still 10-fold upregulated compared with that in the DMSO-treated controls (Fig. 4D). This decreased expression of CGB mRNA after treatment with a combination of $\mathrm{H}-89$ and forskolin may be due to the fact that $C G B$ mRNA expression is partly dependent on the PKA pathway of GCM1 and ERVWE1 activation. If this pathway is blocked, this part of $C G B$ mRNA expression is blocked as well and thus results in a reduced expression of $C G B$ mRNA similar to the effect of $\mathrm{H}-89$ on LGALS13 mRNA expression. However, protein data reveal that there must be at least a second, PKA-independent pathway of CGB expression in mononucleated BeWo cells, which keeps treated BeWo cells in the absence of $\mathrm{H}$-CGB protein expression at levels of forskolin-89. Castellucci \& Kaufmann (2006) have proposed a respective model based on protein data from isolated trophoblasts and villous explant cultures. They proposed that there is a sequence of events in the protein expression of CGA, CGB and finally $\mathrm{hPL}$. hCG is expressed directly prior to fusion, CGB directly after fusion and $\mathrm{hPL}$ slightly later. The authors discuss that in the event of delayed fusion or severe damage of the overlying syncytiotrophoblast, mononucleated cytotrophoblasts may express $\boldsymbol{\alpha}-\boldsymbol{\alpha}$ - and CGB as well (Castellucci \& Kaufmann 2006). Regarding CGB, this is in line with the data from our study, where hindrance of syncytial fusion leads to protein expression of CGB in mononucleated BeWo cells.

The significantly decreased mRNA expression of the sub-membranous cytoskeletal protein SPTAN1 in the presence of forskolin was suggested to result from the increased fusion rate (Gauster et al. 2010), as multinucleated cell structures compared with the respective number of single cells show a reduced surface to volume ratio. Thus, less SPTAN1 is required to sustain the sub-membranous cytoskeletal network in large syncytia compared with mononucleated cells.

Another interesting finding was the disparate expression changes of GCM1 and ERVWE1 mRNA in the presence and absence of $\mathrm{H}-89$ and forskolin (Fig. 4A and $\mathrm{B})$. In the cascade of cAMP-induced cell fusion, the gene product of the transcription factor GCM1 is closely linked to ERVWE1, since the latter is a target gene of GCM1. As expected GCM1 mRNA was not affected by blocking of PKA as this enzyme modifies GCM1 by phosphorylation only post-translationally. Inhibition of this phosphorylation process led to a reduced activity of the transcription factor GCM1 followed by a downregulation of ERVWE1 expression. Through downregulation of this classic fusion peptide, fusion was blocked, and mRNA expression of post-fusion proteins such as LGALS13, CGB and ALPP was also downregulated, even though only partly in the case of CGB. Similar to the data on mRNA, LGALS13 protein expression was completely blocked. Interestingly, CGB protein expression remained unaffected.

Immunofluorescence staining with antibodies against the proliferation marker $\mathrm{Ki}-67$ indicates that cells 
which were hindered to undergo fusion via $\mathrm{H}-89$ were still proliferating as shown by $\mathrm{Ki}-67$ staining but also expressed CGB (Fig. 5B and C). This is in contrast to fused BeWo cells that stain positive for CGB but negative for $\mathrm{Ki}-67$. A combination of all these data
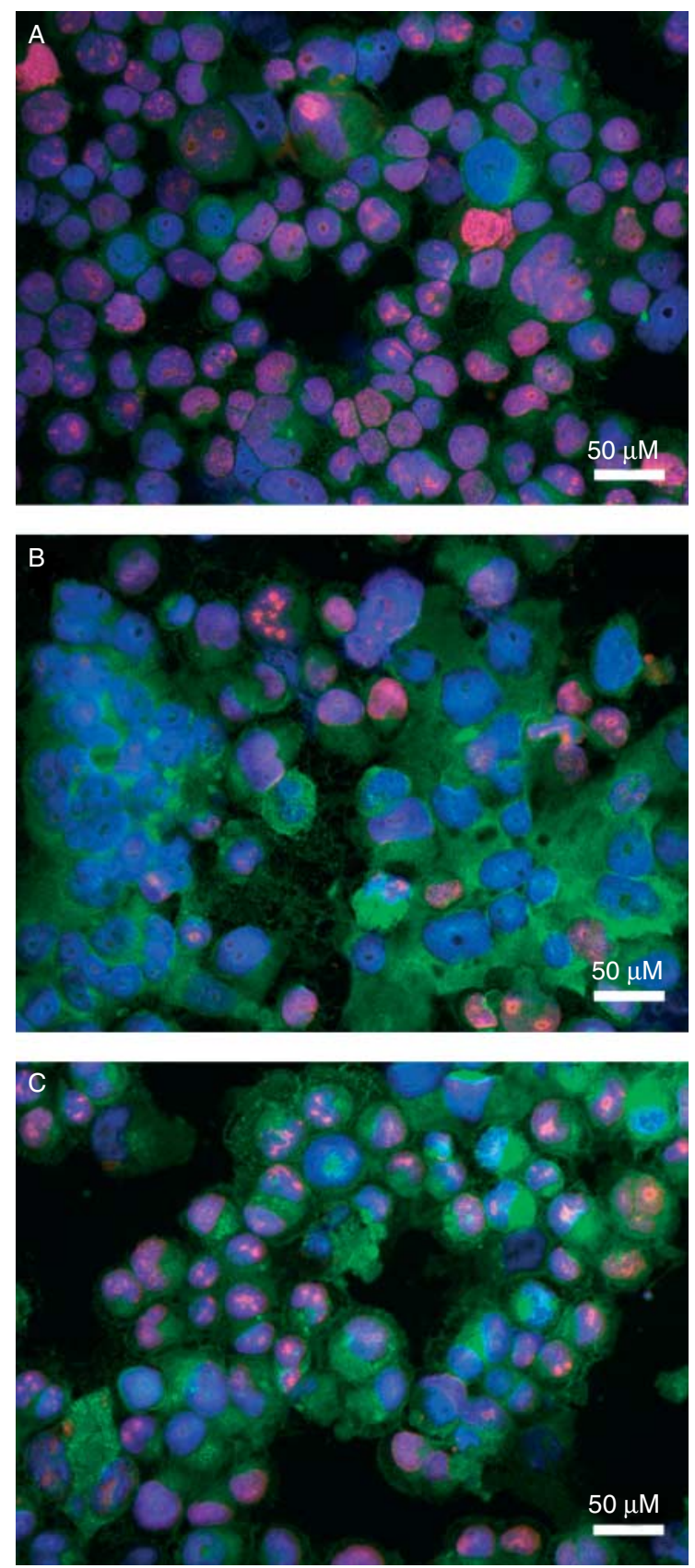

Figure 5 Effect of the protein kinase A inhibitor $\mathrm{H}-89$ on BeWo cell proliferation (48 h). Immunofluorescence staining for $\mathrm{Ki}-67$ (red) and CGB (green) with DAPI counterstaining (blue) was performed on BeWo cytospins as described in Materials and Methods. (A) Control cells treated with DMSO are mostly positive for $\mathrm{Ki}-67$, while (B) cells in the presence of forskolin differentiate into syncytia and stain positive for CGB. (C) In the presence of forskolin and H-89, mononucleated cells maintain their proliferative activity and stain positive for CGB as well. suggests that the forskolin-induced pathway of cell fusion as proposed so far (Fig. 1A) might be more complex, as depicted in Fig. 1B. CGB protein may derive from an additional PKA-independent pathway of CGB expression, which bypasses the regulation of ERVWE1 (Fig. 1B).

These data have important implications for the use of CGB as a marker of syncytialisation of the classical model of trophoblast fusion, the BeWo cells. Any treatment of these cells may lead to a decreased fusion rate without an accompanying decrease in CGB expression. Hence, only measuring CGB alone may not be sufficient to prove fusion of BeWo cells. Instead, it may be valuable to use relative expression of LGALS13, ERVWE1 or even SPTAN1 as additional markers of syncytial fusion of trophoblast and trophoblast-derived cells.

\section{Materials and Methods}

\section{Cell culture}

BeWo cells (European Collection of Cell Cultures, ECACC, Salisbury, UK) were cultured at $37^{\circ} \mathrm{C}$ and $95 \%$ air $/ 5 \% \mathrm{CO}_{2}$ in DMEM (Gibco, Invitrogen) and Ham's F12K medium (Gibco) (ratio 1:1) supplemented with 10\% FCS (PAA, Pasching, Austria), $1 \%$ penicillin/streptomycin (PAA) and $1 \%$ amphotericin B (PAA). Cells were plated in 12-well dishes $\left(2.3 \times 10^{5}\right.$ cells/well) and incubated overnight. The following day, the medium was removed and replaced by fresh medium with or without $20 \mu \mathrm{M}$ forskolin (Sigma-Aldrich) or $0.2 \%$ DMSO (as vehicle for forskolin; Roth/Lactan, Graz, Austria) and incubated for another $48 \mathrm{~h}$.

For morphological analysis, immunofluorescence staining and quantification of fusion, BeWo cells were detached by treatment with $120 \mu \mathrm{l} /$ well Accutase (PAA) supplemented with $0.01 \mathrm{mg} / \mathrm{ml}$ DNase I (Roche) for $15 \mathrm{~min}$ at $37^{\circ} \mathrm{C}$ and spun onto glass slides at $57 \mathrm{~g}$ for $5 \mathrm{~min}$ (Shandon Cytospin 2, Histocom, Vienna, Austria).

\section{Exposure of BeWo cells to $\mathbf{H - 8 9}$ (dihydrochloride)}

The culture medium in the presence of $20 \mu \mathrm{M}$ forskolin or $0.2 \%$ DMSO was supplemented with 1-100 $\mu \mathrm{M}$ dihydrochloride, H-89 (Calbiochem/Merck; Davies et al. 2000). Stock solution of $\mathrm{H}-89$ was prepared in double distilled water. Cells were incubated for $48 \mathrm{~h}$ under the same conditions as described above.

\section{Cell lysis and protein determination}

Cells were washed once in PBS and lysed with $80 \mu \mathrm{l} /$ well RIPA buffer (Sigma-Aldrich; with complete protease inhibitor cocktail, Roche) for $5 \mathrm{~min}$ on ice. Lysates were clarified by centrifugation at $16000 \mathrm{~g}$ for $10 \mathrm{~min}$ at $4{ }^{\circ} \mathrm{C}$. Total protein concentration was assessed by Lowry protein assay. 


\section{DELFIA}

LGALS13 and CGB concentrations of cell lysates were determined using time-resolved fluoroimmunoassay kits according to the manufacturer's manual (PerkinElmer, Turku, Finland). Cell lysates were diluted $1: 3$ and 1:5 in DELFIA diluents I and II respectively. The fluorescent signal of each sample was converted by MultiCalc (PerkinElmer) into the respective protein concentration calculated from a standard curve. LGALS13 and CGB concentrations were normalised to total protein in cell lysates.

\section{Immunofluorescence staining and determination of cell fusion}

Cells detached with Accutase (PAA) and spun on glass slides were fixed in acetone (Merck) for $7 \mathrm{~min}$ prior to staining. UV-Block (Lab-Vision, Thermo Scientific, Vienna, Austria) was supplemented with $10 \%$ human $\mathrm{AB}$-serum and used to block non-specific background staining. Primary antibodies used in this study were directed against CGB $(1: 50$, polyclonal rabbit IgG, cat \# RB-059-A, Thermo Scientific), SPTAN1 (1:100, monoclonal mouse IgG, clone AA6; Biotrend, Cologne, Germany) and Ki-67 (1:100, monoclonal mouse IgG, Mib-1; Dako, Vienna, Austria). Primary antibodies were diluted in antibody diluent (Dako) and incubated for $30 \mathrm{~min}$ at room temperature alone or in combination. Fluorescent-labelled secondary antibodies (Alexa Fluor 555 goat anti-mouse IgG or Alexa Fluor 488 goat anti-rabbit IgG, 1:200; Invitrogen) were incubated for $30 \mathrm{~min}$ at room temperature. Nuclei were counterstained for 5 min with DAPI (1:2000; Invitrogen). Slides were dried, mounted with ProLong Gold antifade reagent (Invitrogen) and analysed by fluorescent microscopy using a Leica DM6000B microscope connected to an Olympus DP72 digital camera (Olympus, Vienna, Austria).

BeWo cell fusion was determined as described previously (Gauster et al. 2010). In brief, BeWo cells were double stained for nuclei (DAPI) and the sub-membranous cytoskeletal protein SPTAN1 to visualise cell borders and the number of nuclei per cell. Analyses were performed in a total of at least 20 randomly selected microscopic fields of each condition, in three independent experiments (newCAST software; Visiopharm, Hoersholm, Denmark). Number of nuclei in syncytia and total number of nuclei were counted, and fusion index was calculated (number of nuclei in syncytia/total number of nuclei $\times 100)$. The fusion index of the forskolin control was set to 1 , and the indices of the other conditions were calculated accordingly.

\section{RNA isolation and real-time RT-PCR}

Total RNA was isolated with TRI Reagent (Applied Biosystems, Vienna, Austria) according to the manufacturer's instructions. The quality of total RNA was assessed by ethidium bromide staining of denaturing agarose gels.

RT of total RNA was performed with high-capacity cDNA RT Kit (Applied Biosystems) according to the manufacturer's manual. In brief, $2 \mu \mathrm{g}$ total RNA of each sample were mixed with the kit components in a total reaction volume of $20 \mu \mathrm{l}$ and incubated for $10 \mathrm{~min}$ at $25^{\circ} \mathrm{C}, 120 \mathrm{~min}$ at $37^{\circ} \mathrm{C}$ and $5 \mathrm{~s}$ at $85^{\circ} \mathrm{C}$ in a thermocycler.

Real-time RT-PCR was performed using the QuantiFast SYBR Green PCR Kit (Qiagen) and QuantiTect Primer Assays (Qiagen) for LGALS13 (Hs_LGALS13_1_SG QuantiTect Primer Assay), CGB (Hs_CGB_1_SG QuantiTect Primer Assay), ERVWE1 (Hs_ERVWE1_1_SG QuantiTect Primer Assay), GCM1 (Hs_Gcm1_1_SG QuantiTect Primer Assay), ALPP (Hs_ALPP_1_SG QuantiTect Primer Assay), SPTAN1 (Hs_SPTAN1_1_SG QuantiTect Primer Assay) and ribosomal protein P0 (Hs_RPLP0_2_SG QuantiTect Primer Assay), the latter being used as internal reference. cDNA $(10.8 \mu \mathrm{l}$ at $20 \mathrm{ng} / \mu \mathrm{l}$ ) was mixed with $2.7 \mu \mathrm{l}$ primers and $13.5 \mu \mathrm{l}$ SYBR Green master mix. Expression levels were analysed in triplicates in a volume of $8 \mu \mathrm{l}$ per well in a 384-well plate (Roche) in a LightCycler 480 (Roche). PCR conditions comprised an initial 5-min denaturating step at $95{ }^{\circ} \mathrm{C}$ and a subsequent two-step cycling including $10 \mathrm{~s}$ at $95^{\circ} \mathrm{C}$ and $30 \mathrm{~s}$ at $60^{\circ} \mathrm{C}$ for 45 cycles. $C_{\mathrm{t}}$ values were automatically generated by the LightCycler 480 Software (Roche), and relative quantification of gene expression was calculated by the standard $\Delta \Delta C_{\mathrm{t}}$ method using the expression of RPLPO as reference.

\section{Statistical analysis}

Real-time RT-PCR and DELFIA data are expressed as mean \pm s.D. Fusion data are presented as box-and-whisker plot. The box represents the interquartile range, which contains $50 \%$ of the values. The whiskers are lines that extend from the box to the highest and lowest values (1.5 times the interquartile distance), excluding outliers. The lower and upper margins of the box indicate the 25th and the 75th percentiles respectively. The median is indicated as a line across the box.

Differences between the treatment conditions were tested with ANOVA or Kruskal-Wallis test, as appropriate, followed by Tukey's post hoc tests. When comparing the control group with each treatment condition, many-to-one testing procedures (Dunn's test, as appropriate) were used. Statistical analysis was performed using SigmaPlot (SigmaPlot 11.0; Systat Software, Erkrath, Germany). $P$ values of $<0.05$ were considered significant.

\section{Declaration of interest}

$\mathrm{H}$ Meiri is CEO and director of Diagnostic Technologies Ltd (DTL) and has options for ordinary shares on the company accounting for $3.5 \%$ in the company shares on a fully diluted basis.

\section{Funding}

This work was partly supported by a research grant of the European Union (FP6 Grant \# 037244, project title Pregenesys) to K Orendi, H Meiri and B Huppertz and by the Franz-LanyarFoundation (Project \# 331) to M Gauster. K Orendi was funded by the EU (FP6 Grant \#037244) within the PhD program Molecular Medicine of the Medical University of Graz. G Moser was funded by the PhD program Molecular Medicine of the Medical University of Graz. 


\section{Acknowledgements}

Thanks to Monika Siwetz and Rudolf Schmied for their valuable help and expertise, and thanks to Gerold Schwantzer for his support in statistical issues.

\section{References}

Butler TM, Elustondo PA, Hannigan GE \& MacPhee DJ 2009 Integrin-linked kinase can facilitate syncytialization and hormonal differentiation of the human trophoblast-derived BeWo cell line. Reproductive Biology and Endocrinology 7 51. (doi:10.1186/1477-7827-7-51)

Castellucci M \& Kaufmann P 2006 Basic structure of the villous trees. In Pathology of the Human Placenta, 5th edn, pp 50-120. Eds K Benirschke, $R$ Baergen \& $P$ Kauffman. New York: Springer.

Davies SP, Reddy H, Caivano M \& Cohen P 2000 Specificity and mechanism of action of some commonly used protein kinase inhibitors. Biochemical Journal 351 95-105. (doi:10.1042/0264-6021:3510095)

Frendo JL, Olivier D, Cheynet V, Blond JL, Bouton O, Vidaud M, Rabreau M, Evain-Brion D \& Mallet F 2003 Direct involvement of HERV-W Env glycoprotein in human trophoblast cell fusion and differentiation. Molecular and Cellular Biology 23 3566-3574. (doi:10. 1128/MCB.23.10.3566-3574.2003)

Gaspard UJ, Hustin J, Reuter AM, Lambotte R \& Franchimont P 1980 Immunofluorescent localization of placental lactogen, chorionic gonadotrophin and its alpha and beta subunits in organ cultures of human placenta. Placenta 1 135-144. (doi:10.1016/S0143-4004(80)80022-1)

Gauster M, Siwetz M, Orendi K, Moser G, Desoye G \& Huppertz B 2010 Caspases rather than calpains mediate remodelling of the fodrin skeleton during human placental trophoblast fusion. Cell Death and Differentiation 17 336-345. (doi:10.1038/cdd.2009.133)

Gerdes J, Schwab U, Lemke H \& Stein H 1983 Production of a mouse monoclonal antibody reactive with a human nuclear antigen associated with cell proliferation. International Journal of Cancer 31 13-20. (doi:10.1002/ijc.2910310104)

Guilbert LJ, Winkler-Lowen B, Sherburne R, Rote NS, Li H \& Morrish DW 2002 Preparation and functional characterization of villous cytotrophoblasts free of syncytial fragments. Placenta 23 175-183. (doi:10.1053/ plac.2001.0756)

Huppertz B, Sammar M, Chefetz I, Neumaier-Wagner P, Bartz C \& Meiri H 2008 Longitudinal determination of serum placental protein 13 during development of preeclampsia. Fetal Diagnosis and Therapy 24 230-236. (doi:10.1159/000151344)

Keryer G, Alsat E, Tasken K \& Evain-Brion D 1998 Cyclic AMP-dependent protein kinases and human trophoblast cell differentiation in vitro. Journal of Cell Science 111 995-1004.

Knerr I, Schubert SW, Wich C, Amann K, Aigner T, Vogler T, Jung R, Dotsch J, Rascher W \& Hashemolhosseini S 2005 Stimulation of GCMa and syncytin via cAMP mediated PKA signaling in human trophoblastic cells under normoxic and hypoxic conditions. FEBS Letters $\mathbf{5 7 9}$ 3991-3998. (doi:10.1016/j.febslet.2005.06.029)

Leemhuis J, Boutillier S, Schmidt G \& Meyer DK 2002 The protein kinase A inhibitor H89 acts on cell morphology by inhibiting Rho kinase. Journal of Pharmacology and Experimental Therapeutics 300 1000-1007. (doi:10.1124/jpet.300.3.1000)
Leisser C, Saleh L, Haider S, Husslein H, Sonderegger S \& Knofler M 2006 Tumour necrosis factor-alpha impairs chorionic gonadotrophin beta-subunit expression and cell fusion of human villous cytotrophoblast. Molecular Human Reproduction 12 601-609. (doi:10.1093/ molehr/gal066)

Lin L, Xu B \& Rote NS 1999 Expression of endogenous retrovirus ERV-3 induces differentiation in BeWo, a choriocarcinoma model of human placental trophoblast. Placenta 20 109-118. (doi:10.1053/ plac.1998.0337)

Lin L, Xu B \& Rote NS 2000 The cellular mechanism by which the human endogenous retrovirus ERV-3 env gene affects proliferation and differentiation in a human placental trophoblast model, BeWo. Placenta 21 73-78. (doi:10.1053/plac.1999.0443)

Mi S, Lee X, Li X, Veldman GM, Finnerty H, Racie L, LaVallie E, Tang XY, Edouard P, Howes S et al. 2000 Syncytin is a captive retroviral envelope protein involved in human placental morphogenesis. Nature $\mathbf{4 0 3}$ 785-789. (doi:10.1038/35001608)

Pidoux G, Gerbaud P, Tsatsaris V, Marpeau O, Ferreira F, Meduri G, Guibourdenche J, Badet J, Evain-Brion D \& Frendo JL 2007 Biochemical characterization and modulation of LH/CG-receptor during human trophoblast differentiation. Journal of Cellular Physiology 212 26-35. (doi:10.1002/jcp.20995)

Schubert SW, Abendroth A, Kilian K, Vogler T, Mayr B, Knerr I \& Hashemolhosseini S 2008 bZIP-type transcription factors CREB and OASIS bind and stimulate the promoter of the mammalian transcription factor $\mathrm{GCMa} / \mathrm{Gcm} 1$ in trophoblast cells. Nucleic Acids Research 36 3834-3846. (doi:10.1093/nar/gkn306)

Spencer K, Cowans NJ, Chefetz I, Tal J \& Meiri H 2007 Firsttrimester maternal serum PP-13, PAPP-A and second-trimester uterine artery Doppler pulsatility index as markers of pre-eclampsia. Ultrasound in Obstetrics \& Gynecology 29 128-134. (doi:10.1002/ uog.3876)

Than NG, Pick E, Bellyei S, Szigeti A, Burger O, Berente Z, Janaky T, Boronkai A, Kliman H, Meiri H et al. 2004 Functional analyses of placental protein 13/galectin-13. European Journal of Biochemistry 271 1065-1078. (doi:10.1111/j.1432-1033.2004.04004.x)

Than NG, Romero R, Goodman M, Weckle A, Xing J, Dong Z, Xu Y, Tarquini F, Szilagyi A, Gal P et al. 2009 A primate subfamily of galectins expressed at the maternal-fetal interface that promote immune cell death. PNAS 106 9731-9736. (doi:10.1073/pnas. 0903568106)

Webb PD \& Todd J 1988 Attachment of human placental-type alkaline phosphatase via phosphatidylinositol to syncytiotrophoblast and tumour cell plasma membranes. European Journal of Biochemistry 172 647-652. (doi:10.1111/j.1432-1033.1988.tb13938.x)

Wice B, Menton D, Geuze H \& Schwartz AL 1990 Modulators of cyclic AMP metabolism induce syncytiotrophoblast formation in vitro. Experimental Cell Research 186 306-316. (doi:10.1016/0014-4827(90) 90310-7)

Received 12 May 2010

First decision 16 June 2010

Accepted 9 August 2010 